\title{
Application of frequency map analysis to beam-beam effects study in crab waist collision scheme
}

\author{
Dmitry Shatilov, Eugene Levichev, and Evgeny Simonov \\ Budker Institute of Nuclear Physics, Novosibirsk, Russia \\ Mikhail Zobov \\ INFN, Laboratori Nazionali di Frascati, Frascati, Italy \\ (Received 5 April 2010; published 21 January 2011)
}

\begin{abstract}
We applied frequency map analysis (FMA) — a method that is widely used to explore dynamics of Hamiltonian systems - to study beam-beam effects in a novel crab waist collision approach. The "crab" focusing of colliding beams results in significant suppression of betatron coupling resonances induced by beam-beam interaction. Application of FMA provides visible information about all working resonances, their widths, and locations in the planes of betatron tunes and betatron amplitudes, so the process of resonances suppression due to the beams crabbing is clearly seen. However, our numerical simulations and further analysis showed that effectiveness of crab waist is considerably restricted in the cases when the colliding beams are not flat. The FMA technique turned out to be very helpful in these studies, as it gave us the key information which would be difficult to obtain in a different way.
\end{abstract}

DOI: 10.1103/PhysRevSTAB.14.014001

PACS numbers: 29.27.Bd

\section{INTRODUCTION}

The crab waist $(\mathrm{CW})$ collision scheme was proposed by Raimondi [1] to enhance the luminosity of electronpositron colliders. The idea of $\mathrm{CW}$ thoroughly examined in $[2,3]$ is, in brief, as follows. Two bunches with small transverse sizes (low emittance beams are essential) intersect at large Piwinski angle, so the length of the overlap area is much smaller than the bunch length. In this case the vertical beta function at IP can be squeezed to the length of the intersection area (submillimeter range) without incurring in the hourglass effect, so the luminosity increases substantially. On the other hand, betatron coupling beambeam resonances are strongly excited in such a scheme, thus limiting the maximum achievable tune shift $\xi_{y}$. This drawback can be overcome by introducing the $\mathrm{CW}$ transformation which is realized by two sextupole magnets placed in phase with the IP in the horizontal plane and at $(2 n+1) \cdot \pi / 2$ in the vertical plane as it is shown in Fig. 1 .

The sextupole strength estimated from optical consideration should be

$$
\frac{B^{\prime \prime} l}{B \rho}=\frac{1}{\theta \beta_{y}^{*} \beta_{y}} \sqrt{\frac{\beta_{x}^{*}}{\beta_{x}}}
$$

where $\theta$ is the full crossing angle and the other parameters are explained in Fig. 1. The sextupoles focus particles locally in such a way that the vertical waist (minimum of $\beta_{y}$ ) rotates and adjusts along the axis of the opposite beam, as it is illustrated in Fig. 2. The CW transformation provides effective suppression of betatron coupling resonances (together with their synchrobetatron satellites), thus increasing the $\xi_{y}$ limit by a factor of about 3. First, it was predicted by simulations and then observed experimentally at the DAФNE $\Phi$ factory $[4,5]$.
Frequency map analysis [6,7] turned out to be very useful for $\mathrm{CW}$ investigations, as it provides visible information about all working resonances, their widths, and locations in the planes of betatron tunes and betatron amplitudes, so the process of resonances suppression due to the beams crabbing is clearly seen. We applied this technique to see how $\mathrm{CW}$ works at the DA $\Phi N E \Phi$ factory, where a very good agreement between simulations and experimental data has been obtained $[8,9]$. Then we performed similar studies for the SuperB project [10].

The success of CW implementation at DA $\Phi$ NE boosted the interest in applying this scheme to other colliders, in particular, the ones with round and quasiround beams, such as LHC. However, our numerical simulations and further analysis showed that effectiveness of $\mathrm{CW}$ is considerably restricted in the cases when the colliding beams are not flat. These results are presented in Sec. V, where the differences between flat and round beams are discussed in the context of CW applicability. The FMA technique played an important role in these studies, as it gave us the key information which would be difficult to obtain in a different way.

\section{FREQUENCY MAP ANALYSIS}

At the phase plot of a Hamiltonian system, one can see a complicated mixture of periodic, quasiperiodic, and

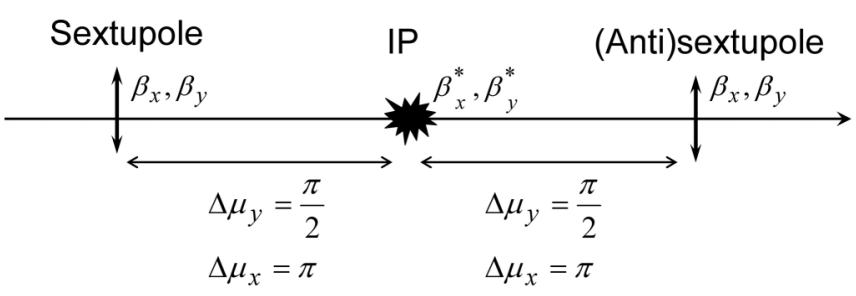

FIG. 1. Crab waist sextupoles location. 

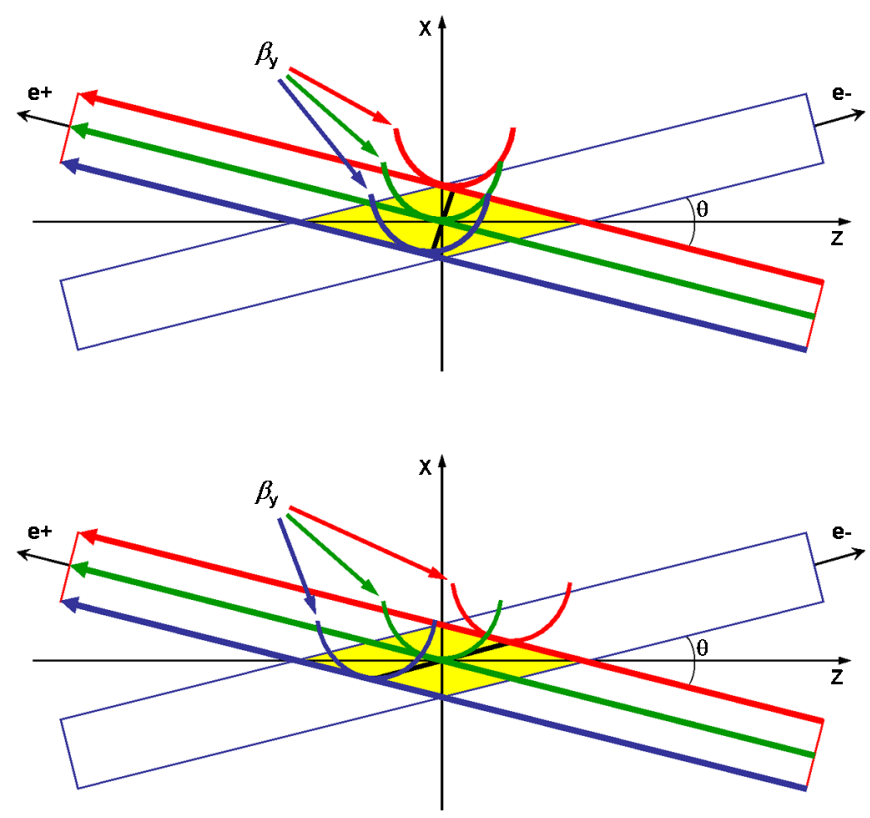

FIG. 2. Vertical beta function at IP without (on top) and with the $\mathrm{CW}$ transformation.

chaotic trajectories arranged in stable and unstable areas. Analysis of these trajectories and distinction between regular (periodic or quasiperiodic) and chaotic ones may give useful information on the motion features. One of the possible techniques providing such info on every particular trajectory is FMA proposed by Laskar [6]. In the accelerator community this method is used mainly for dynamic aperture study, but there are also examples of its successful application to beam-beam effects [11].

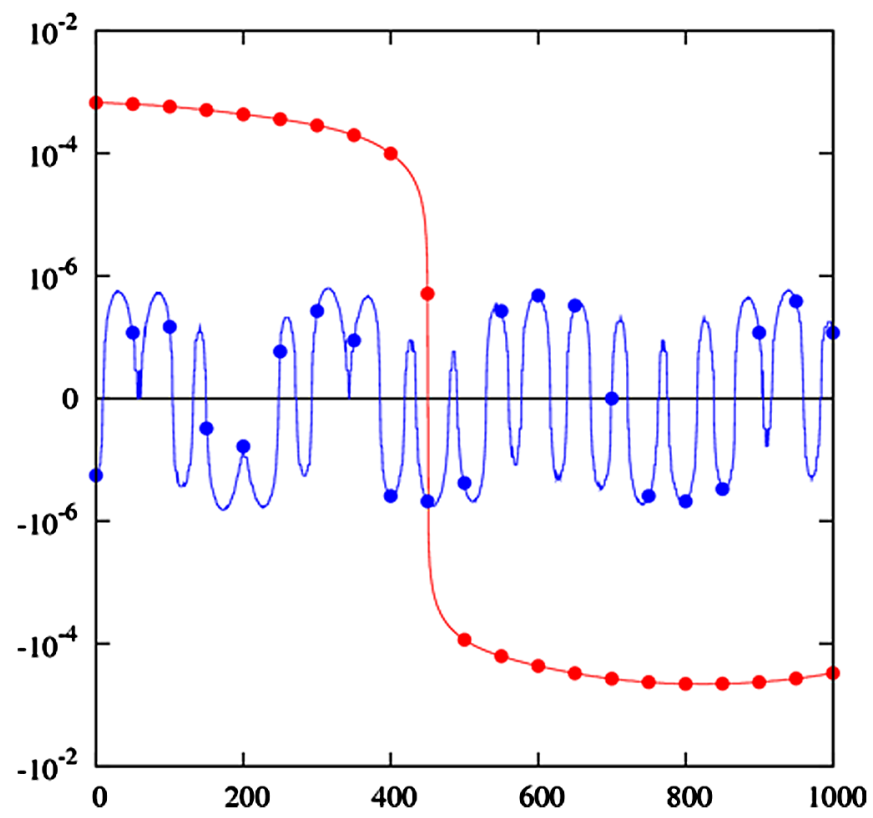

FIG. 3. Betatron tune deviation versus the window shift (in turns) for two trajectories: chaotic (red) and regular (blue).
Since the FMA technique is commonly used, we only briefly mention some peculiarities of its implementation in our studies. For any given initial coordinates, we track a particle for 2024 turns and use the Hanning window of 1024 turns to determine the betatron tunes. Then the window is shifted 20 times by 50 turns, so we get a set of 21 numbers (see Fig. 3, bold dots). The diffusion index is calculated as $\log _{10}\left(\sigma_{\nu}\right)$, where $\sigma_{\nu}$ is the rms spread of tunes.

An example of more detailed time-frequency dependencies for two different trajectories is shown in Fig. 3, where the horizontal axis represents the window shift in turns (bold dots correspond to 50-turn intervals) and the vertical one-the tune deviation from the average in logarithmic scale.

\section{BEAM-BEAM INTERACTION IN DAФNE WITH CRAB WAIST}

The DAФNE $\Phi$ factory was upgraded in the second half of 2007 in order to increase the luminosity and test the crab waist idea [12,13]. As a result the peak luminosity was boosted by a factor of about 3 . The gain could be even larger, but it was limited by the crab sextupoles strength and the effects disturbing positron beam at large currents (e.g. electron cloud instability) $[4,14]$. In order to investigate the innovative $\mathrm{CW}$ collision scheme and find its abilities and limitations due to the beam-beam effects, a "weak-strong" experiment was carried out in May 2009. In our studies we used the machine parameters corresponding to the best luminosity achieved in this experiment, see Table I.

Simulation results of the equilibrium beam density distribution for different crabbings are shown in Fig. 4. Actually, the waist rotation in DAФNE was limited by the strength of crab sextupoles, so it reached only 0.5 of the nominal value while the maximum luminosity is expected at the crab value of about 0.8. On the other hand, without crabbing, not only luminosity drops down significantly but the "weak" bunch dies due to the long tails induced by beam-beam interactions. It means that for the given electron current we can compare the simulation

TABLE I. DAФNE parameters in the "weak-strong" experiment.

\begin{tabular}{lcc}
\hline \hline Horizontal emittance & $\varepsilon_{x}(\mathrm{~cm})$ & $2.50 \times 10^{-5}$ \\
Vertical emittance & $\varepsilon_{y}(\mathrm{~cm})$ & $1.25 \times 10^{-7}$ \\
Horizontal beta function & $\beta_{x}^{*}(\mathrm{~cm})$ & 26.0 \\
Vertical beta function & $\beta_{y}^{*}(\mathrm{~cm})$ & 0.95 \\
Bunch length $\left(e^{-} / e^{+}\right)$ & $\sigma_{z}(\mathrm{~cm})$ & $1.90 / 1.28$ \\
Particles per bunch $\left(e^{-} / e^{+}\right)$ & $N_{p}$ & $(4.075 / 0.342) \times 10^{10}$ \\
Crossing angle & $\theta(\mathrm{mrad})$ & 50 \\
Betatron tunes for $e^{+}$ & $\nu_{x}, \nu_{y}$ & $0.1065,0.1753$ \\
Synchrotron tune & $\nu_{s}$ & 0.01 \\
Tune shifts (tracking) & $\xi_{x}, \xi_{y}$ & $0.0154,0.0894$ \\
\hline \hline
\end{tabular}




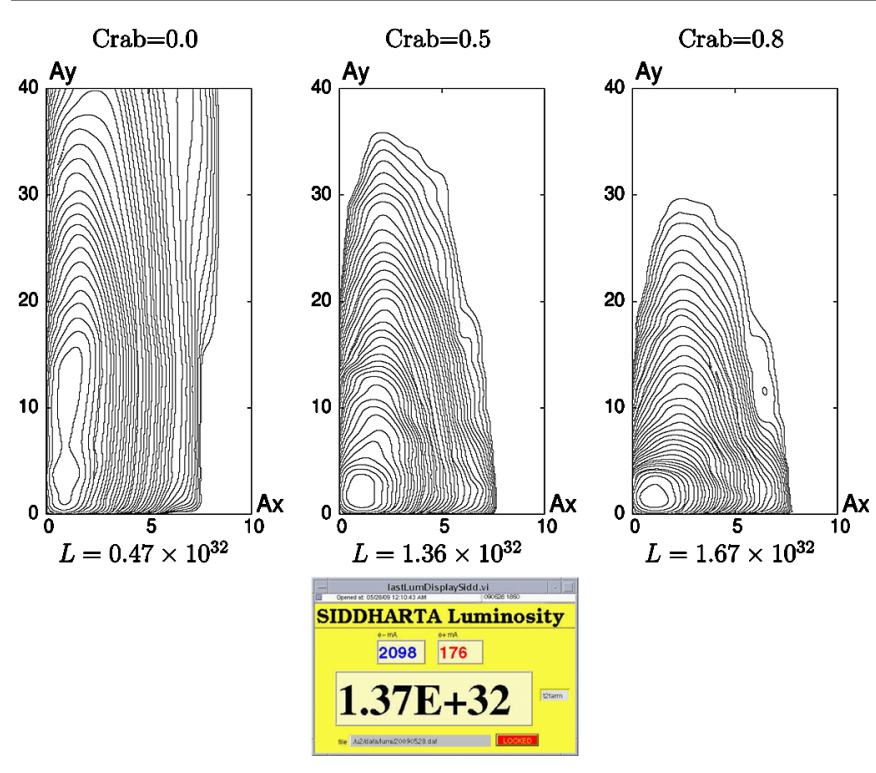

FIG. 4. Contour plots of the equilibrium beam density distribution in the plane of normalized betatron amplitudes and luminosity (numbers at the bottom, in $\mathrm{cm}^{-2} \mathrm{c}^{-1}$ ) versus the crab value. The luminosity obtained experimentally on $\mathrm{DA} \Phi \mathrm{NE}$ with $\mathrm{crab}=0.5$ is shown in the yellow panel.

results with the experimental data only for crab $=0.5$, and the obtained agreement was almost perfect. This gave us more confidence in the tracking code which then was used in our FMA studies.

Usually we build the FMA plots in two planes: betatron tunes and normalized betatron amplitudes. Although the definition of normalized amplitudes for nonlinear trajectories is rather questionable, the information provided by FMA in the amplitude space is useful and interesting, so in order to get these plots we allowed some simplifications. First of all, we use the linear unperturbed (without beambeam) transport matrix to calculate the normalized betatron amplitudes from the physical coordinates. Of course, such amplitudes will not conserve along a trajectory due to nonlinearities, but this is not important for our purposes as we plot the diffusion indexes versus the initial betatron amplitudes. Second, if we examine different betatron phases for the given amplitude, we can get rather different diffusion indexes, so the picture of resonances will be blurred. To avoid this side effect, we examine only one betatron phase for the plots in the amplitude plane, while for the plots in the tune plane many different phases are considered in order to get a more complete picture.

An example of the output data is presented in Fig. 5, where the FMA plots for DAФNE with crab $=0.4$ are shown. Normally we define the trajectory's diffusion index as the maximum of two values calculated from the horizontal and the vertical tune spreads, see Fig. 5(a). In order to estimate the relative contributions of these two components, we also built the plots using only the horizontal tune analysis, see Fig. 5(b). In the latter case the "spectrum" is
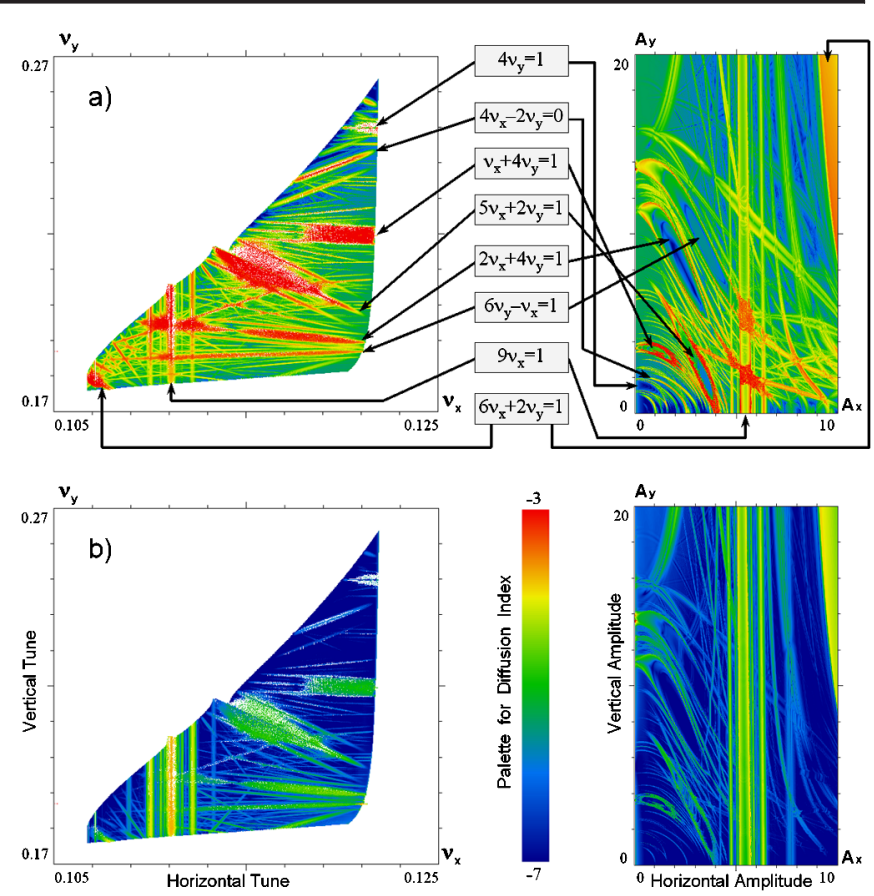

FIG. 5. Beam-beam resonances in the tune and amplitude planes for $\mathrm{DA} \Phi N E$, crab $=0.4$. The diffusion indexes were calculated from both betatron tunes (a), and from the horizontal tune only (b).

distinctly shifted to the blue side (regular motion) in accordance with the well-known feature of flat beams, where the perturbations occur mainly in the vertical plane.

Apparently, many beam-beam resonances including the high-order ones can be clearly seen and identified on both planes. Among them one can distinguish synchrobetatron resonances of different orders - in the plane of tunes they are parallel to the generative betatron ones. It is interesting that, due to the crossing angle, the opposite beam excites small synchrotron oscillations for the particles with zero initial synchrotron amplitudes. Though the excitement is not significant, within $1 \%$ of sigma, it is enough to detect synchrobetatron resonances. But in order to make them more observable we set the initial synchrotron amplitude to 0.1 sigma.

Note how the strong resonances look. In the amplitude plane their widths can be recognized by the red contours corresponding to the separatrix, while in the center of resonance the motion is more regular (green and blue colors). In the plane of tunes strong resonances can be surrounded by specific white areas. Indeed, all trajectories within a resonance island have the same frequencies satisfying the resonance equation, thus they are "attracted" to the resonance line, forming a gap around it. The red points within white areas come from the near-separatrix region, where the tune-amplitude dependence has the largest spread.

The transformations between betatron tunes and betatron amplitudes also are affected by strong resonances, see Fig. 6. The ordinary lines of constant amplitudes in the 

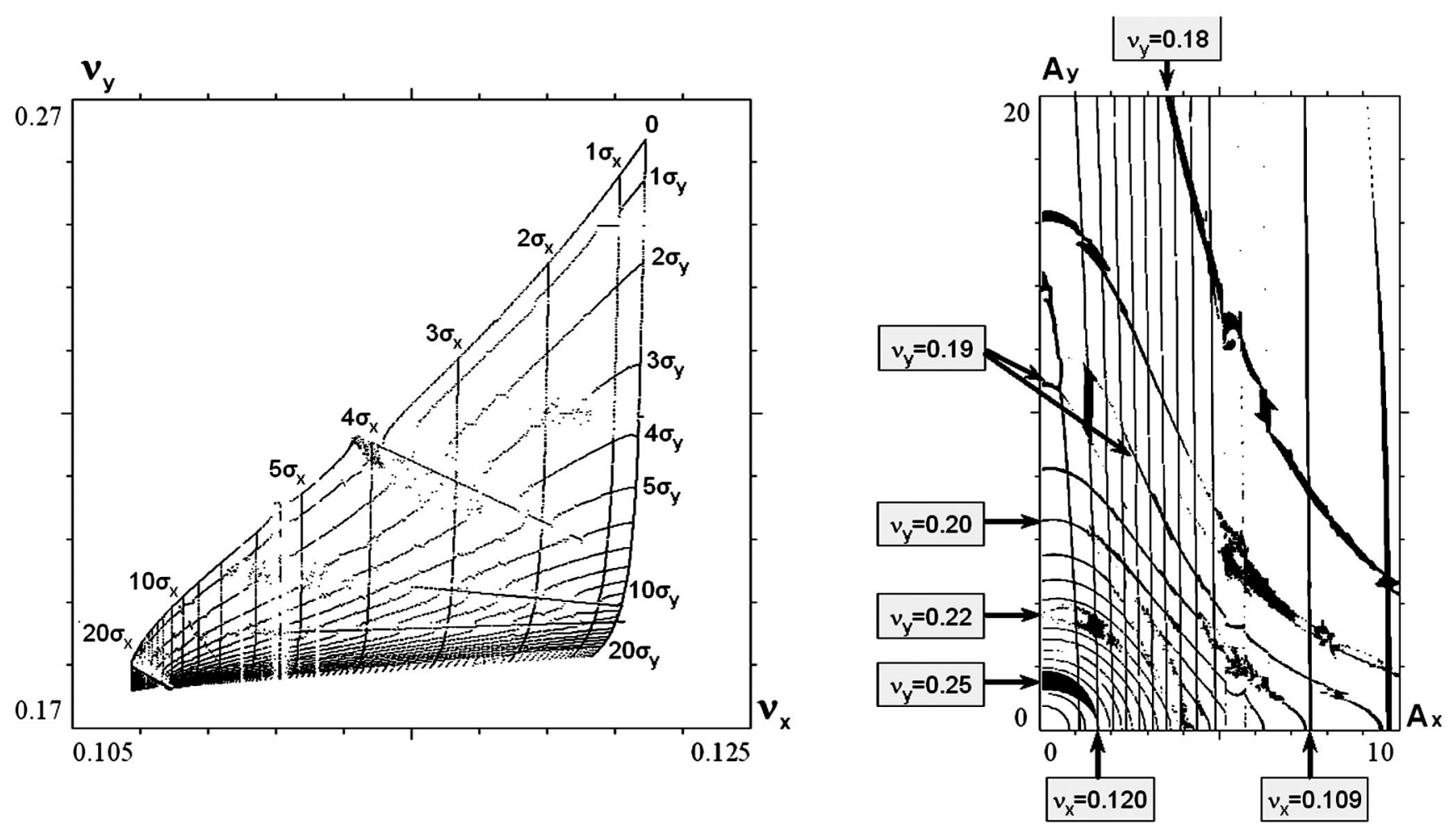

FIG. 6. Transformation between betatron tunes and betatron amplitudes.

footprint (see the plane of tunes) are noticeably disturbed by the trajectories attracted to the resonance lines. The reverse transformation was obtained by the following way. Normally, to produce the FMA plots, we track many particles and scan their initial normalized amplitudes (both horizontal and vertical) with a step of about 0.02 . Then, in order to build the lines of constant tunes in the amplitude plane, we define some allowance around the given values and plot all the points which fall within it. Naturally, the "lines" obtained by this way can be rather thick and shaky, sometimes even noncontinuous. Besides, when they cross strong resonances under small angles, the specific thickenings can be formed-see the right part of Fig. 6.

Good visualization of different resonances and easy estimation of their widths and strengths, provided by the FMA technique, make this method very useful for the crab waist investigations. In order to study how the resonances are suppressed by the $\mathrm{CW}$ transformation, we performed a scan of crab value in the range of 0 to 0.9 (in units of the "nominal") with a step of 0.1, see Figs. 7 and 8. One of the important features facilitating the comparison is that the location of resonances in the tune and amplitude planes is almost not affected. Yet note how the area occupied by the footprint shrinks when the resonances are suppressed.

Actually, we need only the weak beam crabbing to suppress the resonances. But in our simulations, to comply with the reality, we applied the same $\mathrm{CW}$ transformation to both beams. Crabbing of the strong beam makes its distribution essentially non-Gaussian, so the well-known Bassetti-Erskine formulas become nonapplicable for the beam-beam kicks calculations. To solve the problem, a new feature was implemented in the LIFETRAC tracking code, which allowed calculating the kicks from arbitrary beam distributions using the prepared-in-advance grid files [15]. Though the effects of the strong beam crabbing are not significant, some of them have to be mentioned. First, the optimum crab value slightly increases: from 0.6 to about 0.8 . That conforms to the strong-strong simulations by Ohmi [16] and our analytical estimations. Second, the geometrical luminosity increases by a few percents, in accordance with the analytical estimations [17]. Third, the actual tune shifts are also affected: note how the footprint height increases with the beams crabbing.

Using the FMA technique we also have found a good illustration on the well-known Chirikov's criterion of stochasticity. Pay attention to the resonances $2 \nu_{x}+4 \nu_{y}=1$ and $6 \nu_{y}-\nu_{x}=1$ - they are strong enough, close to each other, and isolated from the other strong resonances in some area of their locations. When the beam crabbing decreases from 0.4 to 0.2 , their widths increase and start to overlap, thus creating a stochastic layer in the overlapping region, see the areas indicated by arrows in Fig. 7. The effect is clearly seen on both the tune and the amplitude planes, but the latter one seems to be more relevant as it allows a better detection of the resonance widths. 

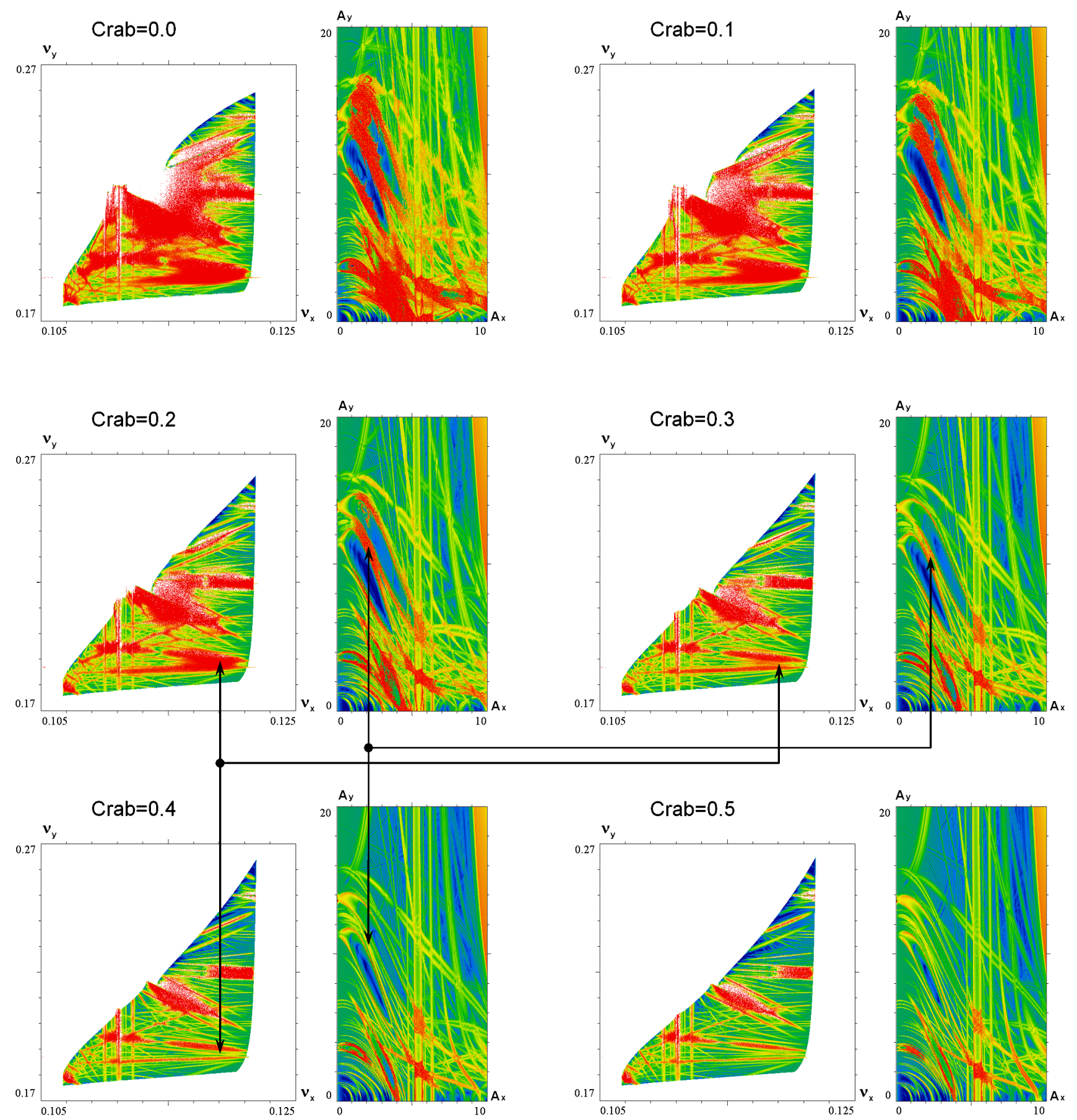

FIG. 7. Beam-beam resonances versus the crab value for DAФNE (part 1).

As it was explained in [2], the optimum angle of waist rotation usually is less than the nominal value. The reason is that, basically, there are two mechanisms exciting beambeam resonances due to the horizontal betatron oscillations. The first and the most important one is the vertical betatron phase modulation, which is suppressed by the nominal $\mathrm{CW}$ transformation $(\mathrm{crab}=1)$. The second is the amplitude modulation of the vertical kick, which can be suppressed only partially, and here the optimum $\mathrm{crab}<1$. When considering both mechanisms, obviously, the optimum must be $<1$, but the actual value can depend on particular resonances, e.g., take a look at the resonances $\nu_{x}+4 \nu_{y}=1$ and $5 \nu_{x}+2 \nu_{y}=1$ indicated by arrows in Fig. 8. Evidently, the optimum crab value is 0.6 for the first one and 0.8 for the second. As for the whole picture, the optimum value lies somewhere between 0.7 and 0.8 . 

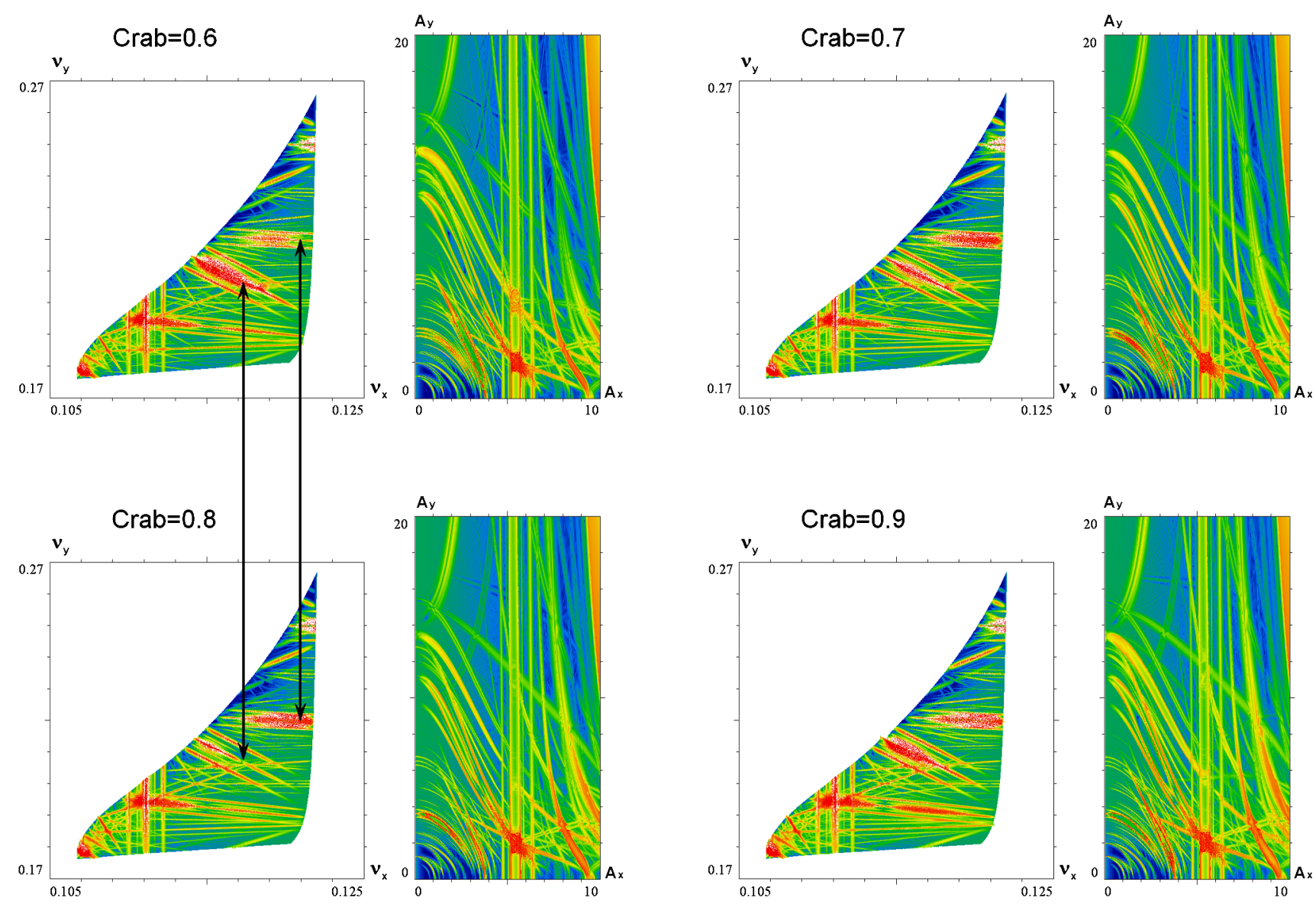

FIG. 8. Beam-beam resonances versus the crab value for DAФNE (part 2).

\section{BEAM-BEAM SIMULATIONS FOR SUPERB}

In the current version of SuperB design [18], there is an asymmetry between HER and LER lattices: emittances and beta functions are different, though the vertical beam sizes at IP are the same. Such asymmetry noticeably affects the beam dynamics. In particular, the hourglass effect and the vertical betatron phase modulation (without $\mathrm{CW}$ ) are much more pronounced in LER [19]. As a result, the beam-beam effects become much stronger for LER regardless of the fact that the nominal tune shifts are the same for both rings. In addition, the optimum crabbings become different too: 0.8 for HER and about 1.0 for LER. On the other hand, the designed beam-beam tune shift $\xi_{y}$ is far below the limit, which is about 0.2 for the SuperB parameters with CW, so we have a rather large margin of safety. This widens our possibilities in choosing the working point, but in any case there is a need in beam-beam simulations, especially for LER which is more vulnerable. Machine parameters used in our studies are presented in Table II.

It is worth mentioning here that the main advantage of crab waist is that it allows an essential increase of the beambeam tune shift. In other words, the effect of $\mathrm{CW}$ becomes valuable only for large $\xi_{y}$ (high bunch currents) and it decreases when $\xi_{y}$ is getting relatively small. The latter was confirmed once again by our simulations, see Fig. 9 . Though the optimum there can be determined, both the luminosity and the beam tails remain almost the same for crab value in the range of 0.8 to 1.0. However, in FMA plots the differences look much more pronounced, see Fig. 10.

In addition, some information can be obtained from the footprints (see the plane of tunes in Fig. 10) even without FMA. First of all, note the footprint shape-how it differs from the "classical" one and how strongly it depends on the crabbing. One more observation is connected with the

TABLE II. The main SuperB parameters (LER/HER), as of September 2009.

\begin{tabular}{lcc}
\hline \hline Energy & $E(\mathrm{GeV})$ & $4.18 / 6.7$ \\
Horizontal emittance & $\varepsilon_{x}(\mathrm{~cm})$ & $(2.56 / 1.6) \times 10^{-7}$ \\
Vertical emittance & $\varepsilon_{y}(\mathrm{~cm})$ & $(6.4 / 4.0) \times 10^{-10}$ \\
Horizontal beta function & $\beta_{x}^{*}(\mathrm{~cm})$ & $3.2 / 2.0$ \\
Vertical beta function & $\beta_{y}^{*}(\mathrm{~cm})$ & $0.02 / 0.032$ \\
Bunch length & $\sigma_{z}(\mathrm{~cm})$ & 0.5 \\
Particles per bunch & $N_{p}$ & $5.74 \times 10^{10}$ \\
Crossing angle & $\theta(\mathrm{mrad})$ & 60 \\
Betatron tunes & $\nu_{x}, \nu_{y}$ & $0.542,0.58$ \\
Synchrotron tune & $\nu_{s}$ & 0.01 \\
Tune shifts (tracking) & $\xi_{x}, \xi_{y}$ & $0.0047,0.1063$ \\
\hline \hline
\end{tabular}



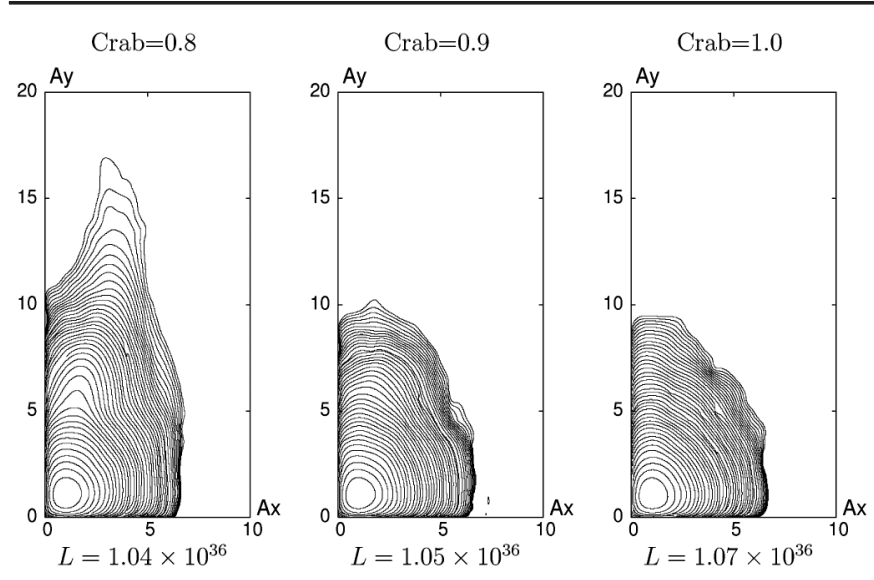

FIG. 9. Contour plots of the equilibrium beam density distribution for SuperB LER and luminosity (numbers at the bottom, in $\mathrm{cm}^{-2} \mathrm{c}^{-1}$ ) versus the crab value.

horizontal tune spread in the beams. As it is seen in Fig. 9, the equilibrium beam distribution is located well within $7 \sigma_{x} \times 10 \sigma_{y}$. Looking at the footprint for $\mathrm{crab}=0.9$, where the lines corresponding to these betatron amplitudes are shown, we may conclude that the actual spread of $\nu_{x}$ is not larger than 0.0003 . That is about $1 / 15$ of the horizontal tune shift $\xi_{x}$, which is also very small itself. This can be considered as one more positive feature of a colliding scheme with large Piwinski angle.

In order to make the FMA plots more informative and allow more resonances to be identified, we enlarged the plotting area to 20 sigma in both directions, while the actual beam density occupies only a small part (in the plane of tunes-close to the "peak"). As we see, the main differences are located at larger amplitudes, that is why they are not seen in Fig. 9.

Based on the results presented in the last two sections, we can compare the FMA technique with the ordinary tracking aimed to simulate the luminosity and equilibrium beam density distribution. Since both methods utilize the same tracking code, they are interconsistent and cannot check each other. The main differences are connected with the radiation damping and noises, which must be taken into account when simulating the equilibrium distribution. Naturally, this complicates identification of resonances in the density contour plots: only strong isolated resonances sometimes can be detected. On the contrary, tracking for FMA must be without noise and damping. This results in a very high resolution of resonances: even high-order ones can be clearly identified. Therefore, FMA can be very useful for investigating particular resonances, their strengths, widths, locations, and dependence on different conditions. On the other hand, tracking for FMA is usually more time consuming (more particle turns are required), and it cannot give the values of luminosity and density in the beam tails (lifetime). Thereby we come to the conclusion that these two techniques are mutually complementary and we should use both of them, as they answer different questions.

Proton and ion colliders, where the radiation effects are negligible, require a separate consideration. Because of the absence of radiation damping, the beam distribution is permanently changing and never attains the equilibrium. However, some specific noises (e.g. intrabeam scattering, ground vibrations, etc.) exist and play an important role in forming the beam density distribution. In addition, it is important that the characteristic time here increases from tens of milliseconds (usual damping time in $e^{+} e^{-}$colliders) to a few hours (luminosity lifetime in proton/ion colliders). In these conditions the high-order resonances become even more crucial. Simulation of the beam density distribution during such a long time will require a huge number of particle turns, and usually it allows only to detect the problem (e.g. increased rate of beam degradation) rather than determine the specific source. Apparently, the FMA technique can be very beneficial here, as it produces a clear picture of resonances within a comparable or even smaller simulation time.

\section{CRAB WAIST FOR [QUASI]ROUND BEAMS}

The success of CW implementation at DA $\Phi$ NE boosted the interest in applying this scheme to other colliders, in particular, the ones with round and quasiround beams. However, it turned out that this feature considerably restricts the effectiveness of $\mathrm{CW}$, if not makes its implementation useless. In this section we discuss the differences between flat and round beams in the context of CW applicability. We start from studying one of the possible schemes of the LHC upgrade, and then consider more general cases to confirm our inferences.

\section{A. Crab waist for the LHC upgrade}

As the base point, we took the IP parameters which can be provided by the optics recently proposed by Fartoukh for the LHC upgrade [20]. However, only for the sake of our studies, we made some minor changes. In particular, the bunch was lengthened and we kept the nominal crossing angle (both values reported in [21]). In this way all the basic CW requirements are practically satisfied: (i) The Piwinski angle $\phi$ is larger than 1 ( $\phi=1.5$, to be compared with $\phi=1.7$ in DAФNE at the maximum luminosity). (ii) The vertical beta function at IP is comparable with the beams overlap area (the ratio $\beta_{y}^{*} / \sigma_{z}$ is similar to that in DAФNE operating conditions). The beam sizes aspect ratio at IP is $\sigma_{x}^{*} / \sigma_{y}^{*}=2$, so the beams are though not round, but "almost" round-in the sense of "not flat." In order to exploit fully the crab waist potential, we initially considered the maximum bunch intensity which is thought to be achievable in LHC [22]: $N_{p}=4.0 \times 10^{11}$. However, LHC operates with two IPs on the ring, and the betatron phase advances between them do matter. Since our studies 

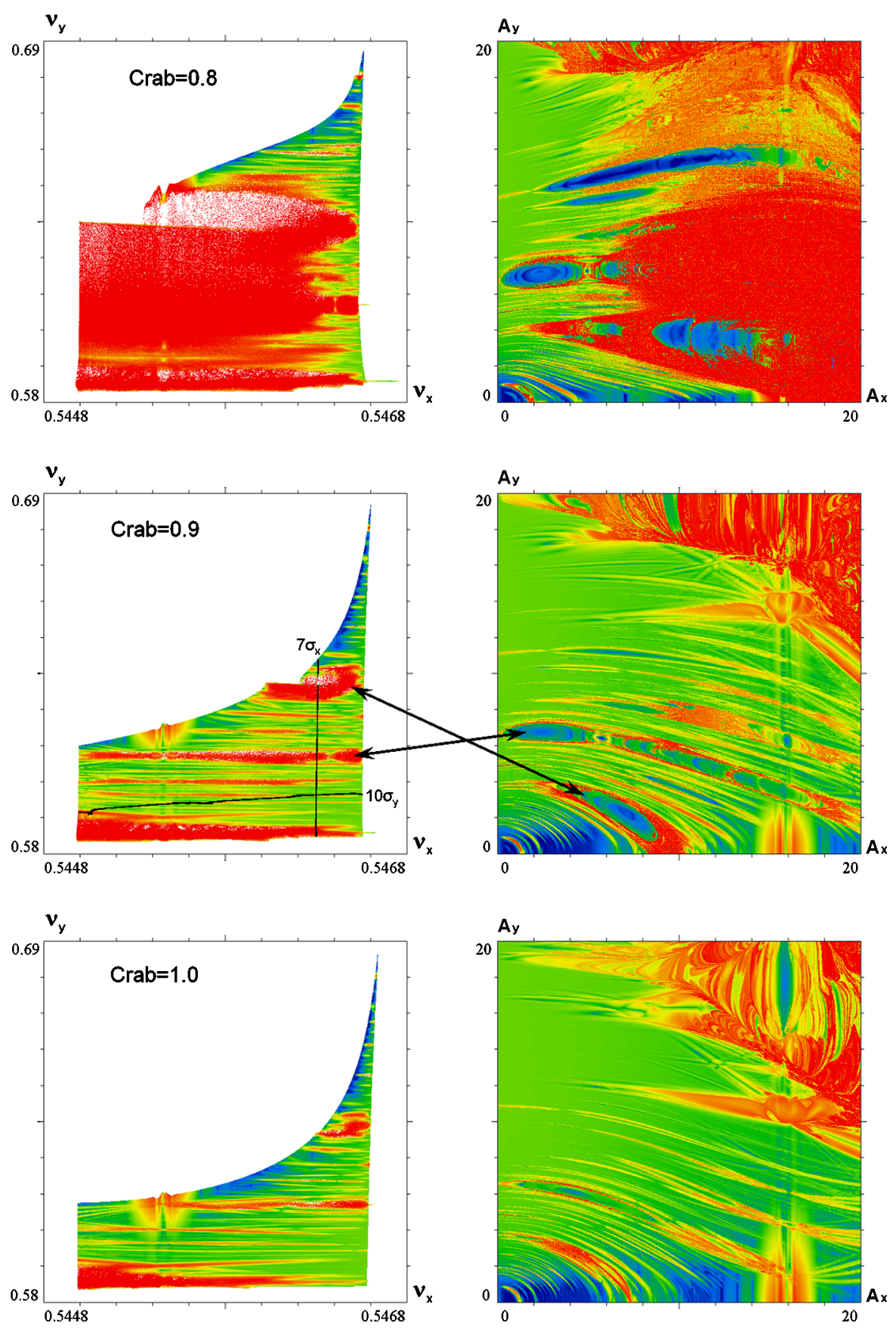

FIG. 10. Beam-beam resonances versus the crab value for SuperB LER.

were restricted to the $\mathrm{CW}$ applicability, we simplified the task and considered only one IP. Accordingly, the bunch intensity was doubled in order to have the correct total tune shifts. The betatron and synchrotron tunes are those used in the present LHC operating conditions [22]. See Table III for the full list of parameters in these simulations.

As usual, the crab sextupoles strength was scanned in order to find the optimum. For the sake of comparison, we also considered the crab crossing (CC) scheme. The simulation results are presented in Fig. 11. Naturally, the tune shifts are negative as both beams consist of protons. As one
TABLE III. LHC parameters used in crab waist simulations.

\begin{tabular}{lcc}
\hline \hline Energy & $E(\mathrm{TeV})$ & 7 \\
Emittances & $\varepsilon_{x, y}(\mathrm{~cm})$ & $5.03 \times 10^{-8}$ \\
Horizontal beta function & $\beta_{x}^{*}(\mathrm{~cm})$ & 30.0 \\
Vertical beta function & $\beta_{y}^{*}(\mathrm{~cm})$ & 7.5 \\
Bunch length & $\sigma_{z}(\mathrm{~cm})$ & 11.8 \\
Particles per bunch & $N_{p}$ & $8.0 \times 10^{11}$ \\
Crossing angle & $\theta(\mathrm{mrad})$ & 0.315 \\
Betatron tunes & $\nu_{x}, \nu_{y}$ & $0.31,0.32$ \\
Synchrotron tune & $\nu_{s}$ & 0.002 \\
\hline \hline
\end{tabular}



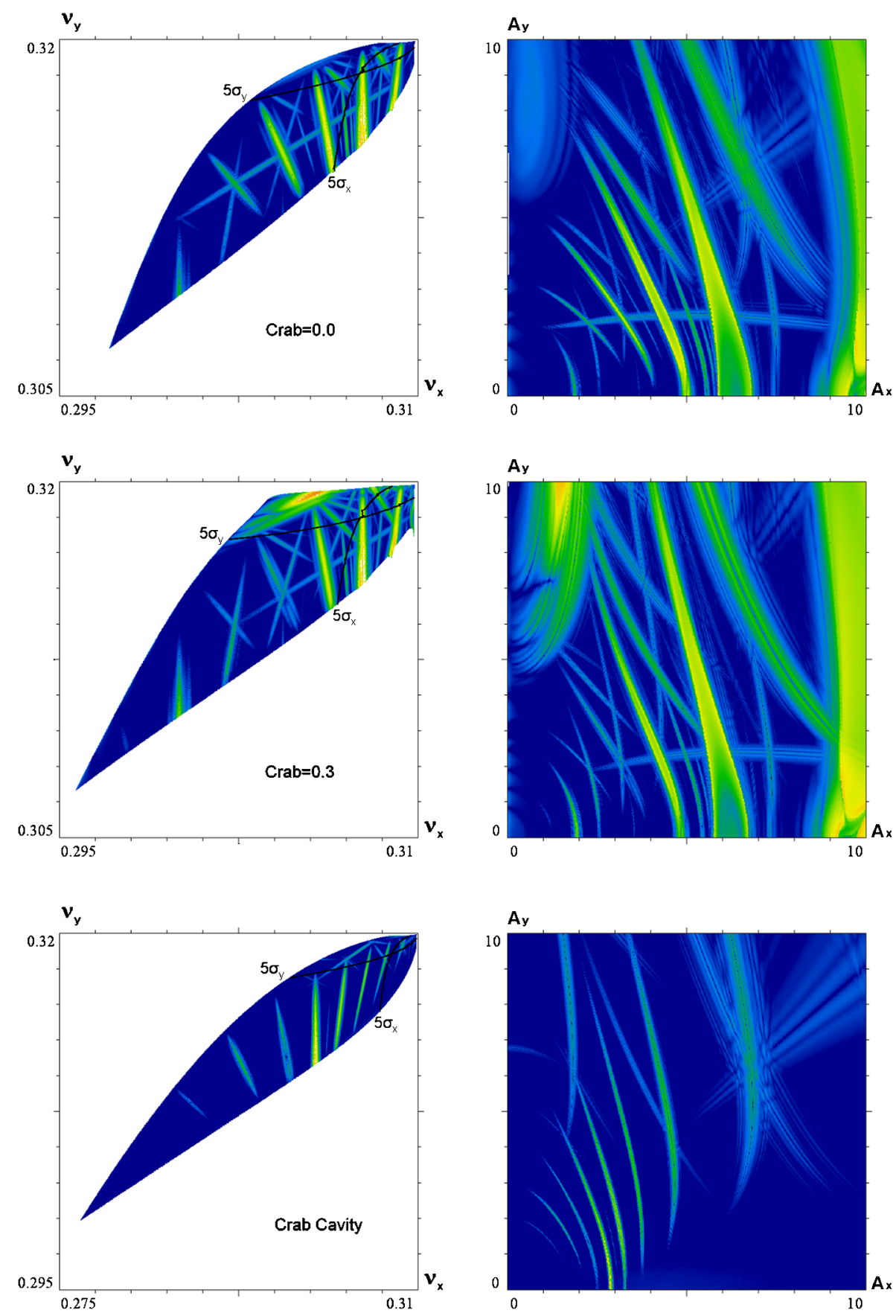

FIG. 11. FMA plots for LHC upgrade in the planes of betatron tunes (left) and normalized betatron amplitudes (right). The case without crabbing is shown on top, the optimum crab waist - in the middle, and crab crossing scheme-at the bottom. Black lines in the plane of tunes correspond to the normalized betatron amplitudes of 5 sigma.

can see, the beam-beam resonances are almost not affected by the crab waist, but can be effectively suppressed by the crab crossing. Detailed inspection shows that $\mathrm{CW}$ makes some slight improvements in the core region (small amplitudes), but in the tails (large amplitudes) it has a negative impact and besides widens the footprint. Note also that the tune shifts are larger for the CC scheme, especially the horizontal one, so the scales in the plane of tunes are different in Fig. 11. Obviously, the luminosity will be also higher for the $\mathrm{CC}$ scheme due to the geometrical factor (the overlap area increase), but we do not discuss here the luminosity and will concentrate on the resonances suppression only.

\section{B. Why does CW not work for round beams?}

In our opinion, the main reason which makes the $\mathrm{CW}$ impact on flat and [quasi]round beams so different can be explained as follows. First, let us consider the flat beams. 
The kick which a test particle (red star in Fig. 12) experiences from the opposite bunch is indicated by vector $\overrightarrow{\boldsymbol{F}}$, and its vertical component obviously is proportional to $\sin (\alpha)$. When the opposite beam is separated horizontally by $\sim \sigma_{x}$ or larger (e.g. due to a crossing angle, when the test particle is close to IP, but shifted longitudinally by a distance comparable with $\sigma_{z}$ ), the vertical component of the kick becomes small since the angle $\alpha$ can be estimated as $\sigma_{y} / \sigma_{x} \ll 1$.

It means that the vertical kicks actually come only from a small part of the opposite beam, in the vicinity of the point where the test particle crosses its longitudinal axis. In [2] this feature was reflected by the scheme shown in Fig. 13. A proper consideration of the opposite bunch implies that it is represented by a number of slices, so the test particle sequentially interacts with each slice when it crosses its plane. However, for simplified models (not for tracking code, of course) sometimes it is convenient to reduce the particle-bunch interaction to a single kick. Thus, we come to a definition of the so-called collision point (CP) - the point where an "integrated" kick occurs. Normally, it is the point where the test particle crosses the central slice of the opposite bunch. The situation becomes more complicated in the collision scheme we are considering now. Namely, the horizontal and the vertical integrated kicks occur in the different points. So, the $\mathrm{CP}$ remains standard for the horizontal kick, but for the vertical one (which is more important for the flat beams) it should be redefined as the point where the particle crosses the longitudinal axis of the opposite beam. An illustration is shown in Fig. 13, where the test particle is shifted longitudinally and horizontally from the bunch center by $Z$ and $X$, respectively, and its $C P$ is marked by a small red star.

The positive effect of this approach is that the $X$ coordinate of CP in the opposite bunch's coordinate system is always zero, which could result in suppression of many resonances. But instead, the new strong effect appears: due

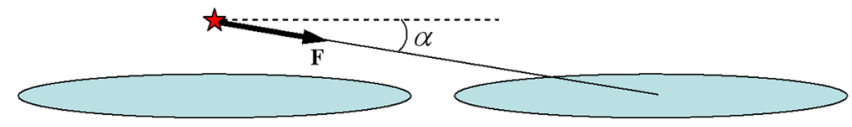

FIG. 12. Kick from a flat beam separated horizontally.

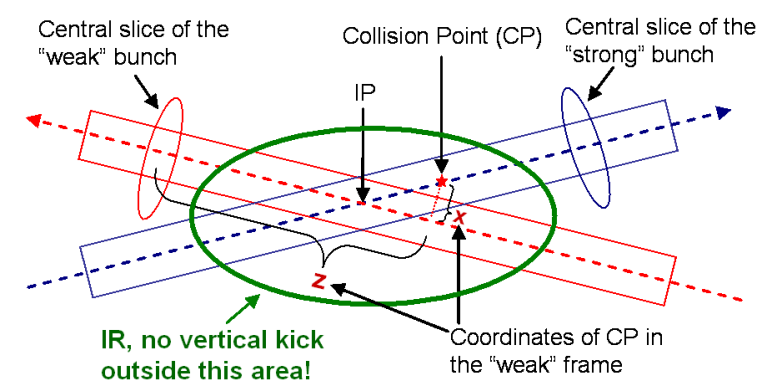

FIG. 13. Collision scheme with large $\phi$ (flat beams). to the horizontal betatron oscillations (i.e. when $X \neq 0$ ), the CP shifts longitudinally from IP. This, in turn, results in the vertical betatron phase modulation at $\mathrm{CP}$, thus exciting the $X-Y$ coupling resonances. In other words, the mechanisms of exciting the resonances are different for the "old-style" and "new-style" CPs. Then the crab waist enters the game, eliminating the betatron phase modulation and thereby suppressing the resonances.

For nonflat beams, on the contrary, the situation is rather different: since $\sigma_{y}$ is comparable with $\sigma_{x}$, the angle $\alpha$ is not small and particles feel the vertical kicks from the opposite bunch even when separated horizontally by a few $\sigma_{x}$. In this case the new definition of $\mathrm{CP}$ becomes somehow irrelevant, and we have to switch to the old one. So, what we finally have for the round beams and not too large Piwinski angles: (i) Vertical betatron phase modulations at $\mathrm{CP}$ due to the horizontal betatron oscillations disappear, since $\mathrm{CP}$ is now "old style." (ii) Therefore, there is no need in CW. However, if applied, it still kills the vertical betatron phase modulation at the "new-style" CP (that is not important now) and introduces additional modulation at the "oldstyle" CP, which is more relevant for the round beams. (iii) The resonances, which normally exist in the schemes with crossing angle, are not suppressed.

Of course, this is a simplified schematic view, and the reality is more complicated. But in general, the simulation results are in agreement with this model. In particular, note that in the regions of small betatron amplitudes $\alpha$ remains small even for the round beams, that is why $\mathrm{CW}$ somehow works there; and the impact of $\mathrm{CW}$ becomes negative at large amplitudes-where $\alpha$ is large. So we may conclude that, in order to make CW effective, it is necessary to have $\alpha \ll 1$ for the particles shifted longitudinally from IP by a distance comparable with $\sigma_{z}$. For flat beams this requirement is met when the Piwinski angle $\phi>1$. And, probably, for round beams CW also should work for very large Piwinski angles.

\section{Effectiveness of CW: From round to flat beams}

In order to justify the explanations given above and to improve our general understanding of $\mathrm{CW}$, we performed additional simulations. Now we are only interested in how the principle works, so there is no necessity to keep the LHC parameters. So as to magnify the effects we intentionally increased the bunch intensity by a factor of 2 ; in this way more beam-beam resonances cross the footprint. The working point also has been changed to (0.307, 0.340) - farther from the main coupling resonance.

For transition from round to flat beams, we need to change some parameters. The simplest way for that would be a mere decrease of the vertical emittance, but this also implies decreasing the bunch current in order to keep $\xi_{y}$ constant, and therefore results in $\xi_{x}$ decrease. In order to keep both footprint sizes more or less unchanged, we performed the transition in a special way. Our goal is to 
change the $\sigma_{x} / \sigma_{y}$ ratio while keeping the same Piwinski angle, vertical beta-function and bunch length (to have the same hourglass), and the tune shifts- to see the same set of resonances in all cases. This can be done if the product of $\sigma_{x} \times \sigma_{y}$ is not changed and the crossing angle is not fixed. So, what we do to increase the $\sigma_{x} / \sigma_{y}$ ratio by a factor of $M$ is the following: (i) decrease the vertical emittance $\varepsilon_{y}$ by
$M$; (ii) increase the horizontal beta function $\beta_{x}$ by $M$; (iii) increase the crossing angle $\theta$ by $\sqrt{M}$.

For these transformations, if the bunch intensity remains constant, the luminosity will almost not change as well. However, to keep the vertical tune shift $\xi_{y}$ exactly the same for all cases, the bunch current was slightly adjusted. We examined the variants with $M$ equal to 1,3 , and 10 , which
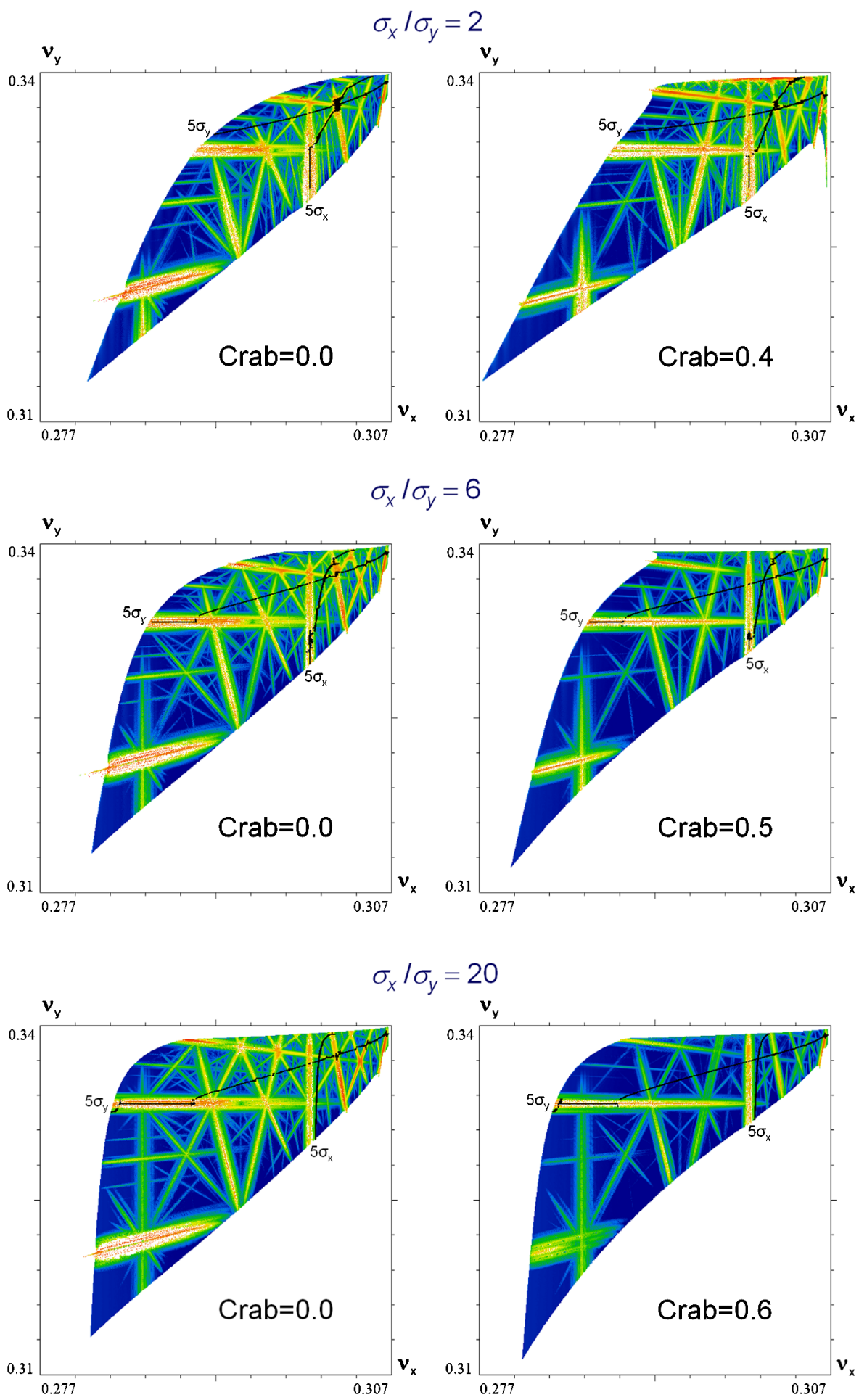

FIG. 14. Efficiency of CW versus the $\sigma_{x} / \sigma_{y}$ ratio. 


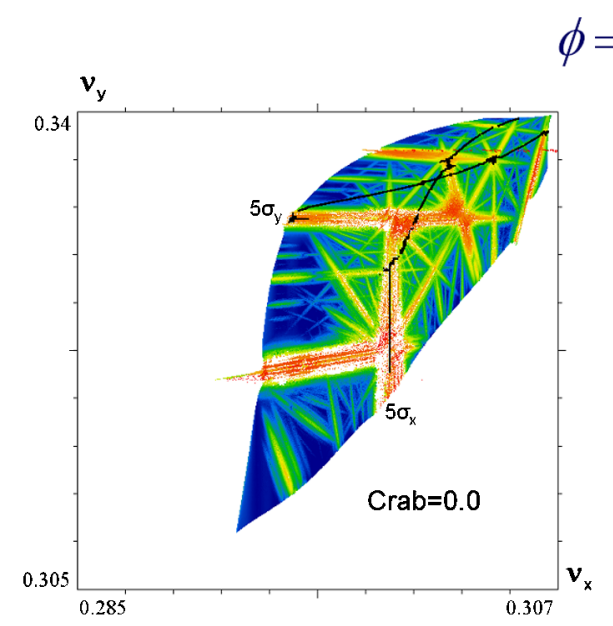

$$
\phi=3
$$
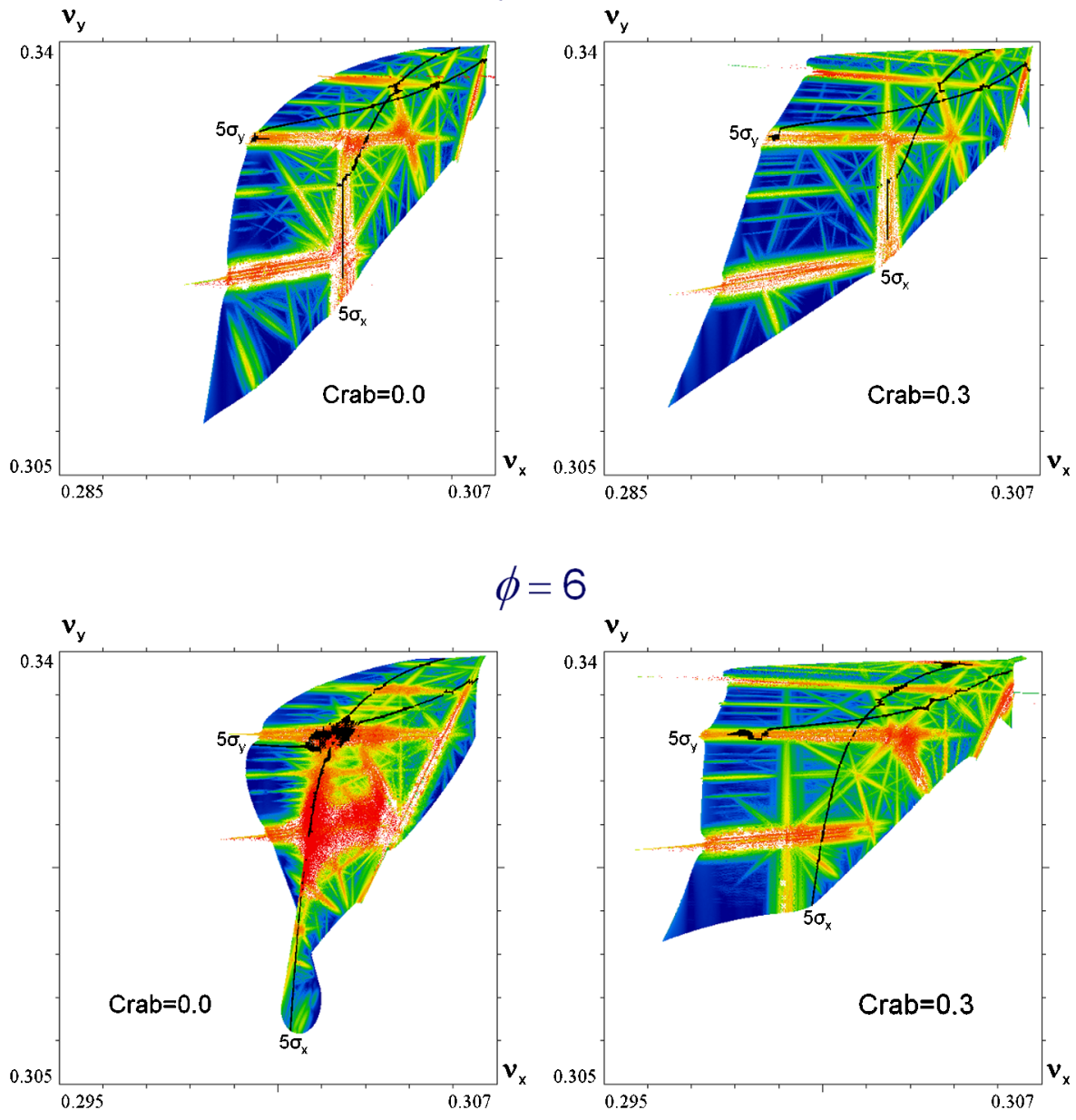

FIG. 15. Efficiency of CW versus the Piwinski angle for $\sigma_{x} / \sigma_{y}=2$.

correspond to the $\sigma_{x} / \sigma_{y}$ ratio (parameter of ellipticity) of 2,6 , and 20, respectively. For each case we performed a $\mathrm{CW}$ scan and compare the results of $\mathrm{CW}=0$ and $\mathrm{CW}=$ optimum (see Fig. 14). As it is seen, for higher bunch intensities the CW has a notable positive impact even for almost round beams, but mainly at small betatron amplitudes, while at large amplitudes the impact is more neutral or even negative and the footprint widens. When the beam is getting more flat, $\mathrm{CW}$ starts working at large amplitudes as well, and the footprint area even shrinks. Note also that the optimum crab strength increases and gets closer to the nominal; this is one more indication that $\mathrm{CW}$ functions better for flat beams. Judging by these results, we need $\sigma_{x} / \sigma_{y} \geq 10$ to make $\mathrm{CW}$ working in full strength.

\section{Crab waist for round beams: Dependence on $\phi$}

Another possibility to decrease the angle $\alpha$ (see Fig. 12) is that of increasing the horizontal separation between two bunches, i.e., increasing the Piwinski angle $\phi$. In simulations we did this simply by changing the bunch length. Of course, such a transformation affects the tune shifts and, what is more important, the $\xi_{x} / \xi_{y}$ ratio. Simulation results for $\phi=3$ and 6 are shown in Fig. 15. Note also that the "base" case of $\phi=1.5$ is shown in Fig. 14 (on top), and the horizontal scales for all three cases are different.

In order to have a similar set of resonances crossing the footprint in all cases, we adjusted the bunch currents to get the same vertical tune shift for the particles with small amplitudes. As it is seen, for $\phi=6$ and $\mathrm{CW}=0$ there is a footprint folding. Interestingly, the $\mathrm{CW}$ transformation recovers the normal footprint shape. In general, due to the large differences in the footprint shapes and sizes, these comparisons are inferior in quality compared to Fig. 14. But anyway it is clear that, even for almost round beams, $\mathrm{CW}$ works better when $\phi$ increases.

\section{CONCLUSION}

The capability of investigating every particular resonance, provided by FMA, is very helpful for a better understanding of various mechanisms affecting the nonlinear beam dynamics. Using this technique, we obtained interesting information about crab waist collisions; demonstration of how CW works for flat beams is clear and impressive. 
For round and quasiround beams, $\mathrm{CW}$ produces a positive effect only at small betatron amplitudes, while at large amplitudes the impact can be even negative. According to our simulations, $\mathrm{CW}$ in general works well for $\sigma_{x} / \sigma_{y} \geq 5$, and in full strength-for $\sigma_{x} / \sigma_{y} \geq 10$. Increasing the Piwinski angle also facilitates the $\mathrm{CW}$ utilization for nonflat beams.

\section{ACKNOWLEDGMENTS}

The BINP authors greatly appreciate the long-lasting fruitful collaboration with the DAФNE team, whose remarkable achievements inspired them to initiate these studies. All simulations were performed on the computing cluster of Novosibirsk State Technical University, Department of Computer Engineering. The authors would like to thank the department's staff for the provided resources and technical support.

[1] P. Raimondi, in the 2nd Workshop on Super B-Factory, Frascati, 2006 [http://www.lnf.infn.it/conference/superb06/ talks/raimondi1.ppt].

[2] P. Raimondi, D. Shatilov, and M. Zobov, arXiv:physics/ 0702033.

[3] P. Raimondi, M. Zobov, and D. Shatilov, in Proceedings of the 11th European Particle Accelerator Conference, Genoa, 2008 (EPS-AG, Genoa, Italy, 2008), pp. 26202622.
[4] C. Milardi et al., ICFA Beam Dyn. Newslett. 48, 23 (2009).

[5] M. Zobov et al., Phys. Rev. Lett. 104, 174801 (2010).

[6] J. Laskar, Icarus 88, 266 (1990).

[7] J. Laskar, in Proceedings of the 20th Particle Accelerator Conference, Portland, OR, 2003 (IEEE, New York, 2003), p. 378.

[8] P. Piminov et al., in Proceedings of the 23rd Particle Accelerator Conference, Vancouver, Canada, 2009 (IEEE, Piscataway, NJ, 2009), wE6PFP049.

[9] M. Zobov et al., ICFA Beam Dyn. Newslett. 48, 34 (2009).

[10] M. Bona et al., arXiv:0709.0451.

[11] Y. Papaphilippou and F. Zimmermann, Phys. Rev. ST Accel. Beams 2, 104001 (1999).

[12] M. Zobov for DANE Collaboration Team, Phys. Part. Nucl. Lett. 5, 560 (2008).

[13] D. Alesini et al., Nucl. Phys. Proc. Suppl. 385, 181 (2008).

[14] C. Milardi et al., Int. J. Mod. Phys. A 24, 360 (2009).

[15] D. Shatilov, ICFA Beam Dyn. Newslett. 52, 42 (2010).

[16] K. Ohmi (private communication).

[17] I. Koop and D. Shvarts (private communication).

[18] M. Biagini et al., "Design of a $10^{36} \mathrm{~cm}^{-2} \mathrm{~s}^{-1}$ Super-B Factory," EPAC08-WEPP039, Genoa, Italy, 2008.

[19] D. Shatilov, P. Piminov, and M. Zobov, Technical Report No. SB-NOTE-ACC-2008-002, 2008.

[20] S. Fartoukh, "A new pass towards the LHC upgrade using "conventional' hardware," for the LMC (2010).

[21] W. Scandale and F. Zimmermann, Nucl. Phys. B, Proc. Suppl. 207, 177 (2008).

[22] F. Zimmermann (private communication). 